\title{
Multiple feature points representation in target localization of wireless visual sensor networks
}

\author{
Wei Li, Jorge Portilla, Félix Moreno, Guixuan Liang, Teresa Riesgo
}

\begin{abstract}
This paper discusses the target localization problem in wireless visual sensor networks. Additive noises and measurement errors will affect the accuracy of target localization when the visual nodes are equipped with low resolution cameras. In the goal of improving the accuracy of target localization without prior knowledge of the target, each node extracts multiple feature points from images to represent the target at the sensor node level. A statistical method is presented to match the most correlated feature point pair for merging the position information of different sensor nodes at the base station. Besides, in the case that more than one target exists in the field of interest, a scheme for locating multiple targets is provided. Simulation results show that, our proposed method has desirable performance in improving the accuracy of locating single target or multiple targets. Results also show that the proposed method has a better trade off between camera node usage and localization accuracy.
\end{abstract}

\section{Introduction}

In wireless visual sensor networks (WVSNs), sensor nodes that are equipped with cameras have functionalities of capturing visual information about targets and delivering the visual data to a base station for further analysis and decision making. Thus, WVSNs is capable of various security and surveillance applications, such as public security, facilities surveillance and monitoring. For most of these applications, the users are interested not only in existence of targets, but also in the positions of the targets (Liu et al., 2010), because the positions could facilitate target detection, recognition and tracking.

The task of localization provides with coordinates of both sensors and targets in sensor works (Soro and Heinzelman, 2009). Thus, localization task contains self localization of sensor nodes and target localization. In this paper, we focus on the problem of target localization while the locations of sensor are already known. Target localization is to estimate the location of a target in the world coordinate based on the visual information of camera nodes (Kulkarni, 2007). The problem of target localization is well studied in wireless sensor networks. The measurement techniques in sensor localization include angle of arrival (AOA) measurements, distance related measurements and received sig nal strength (RSS) measurements (Mao et al., 2011). The existing techniques of target localization cannot be applied in WVSN. For example, multi target can be cooperatively tracking by the Markov chain Monte Carlo data association method (Jiang and Hu, 2013). However, this method cannot address the problem of target localization in WVSN due to the significant differences in informa tion capturing and processing between visual sensors and binary sensors. Actually, target localization in WVSN faces great chal lenges. Firstly, image processing is in general costly to implement in local nodes (Ercan et al., 2006), because the capabilities of computing are limited in local nodes. Secondly, the bandwidth resources are also restricted in WVSNs. Thus, there are constraints to transmit a huge amount of visual data generated by cameras to a central node or a base station (Charfi et al., 2009). Thirdly, since the sensing capability of a camera is characterized by directional sensing, the location information of a target in the depth dimen sion is lost in an image. Fourthly, due to the cost limitation, visual nodes in WVSN are equipped with low resolution optical sensors (Akyildiz et al., 2007). Thus, the accuracy of filtering and extraction of target's position relevant information cannot be guaranteed in local sensor level.

Vision based surveillance by multiple cameras receives con siderable attentions, since visual surveillance by multiple cameras will enlarge the area and information from multiple views can be used to solve many problems (Liu et al., 2010). For example, the accuracy of the target localization can be gradually improved by 
selecting the most informative cameras based on correlation functions (Dai and Akyildiz, 2009) and the properties of the overlap region of the target in images (Li and Zhang, 2012) until the required accuracy level of target state is achieved. However, the multiple cameras bring new problems. Finding the correlated points pair in different images of cameras is a very difficult task. Furthermore, the energy and wireless channel capacity are very limited in WVSN. As discussed above, WVSN is a kind of resource limited networks in nature. It is desirable to balance the trade off between the accuracy of localization and the resources of WVSN.

The motivation of our study is to use the visual data acquired from the camera nodes to accurately estimate the position of target in the world coordinate. In this paper, we provide a method that uses multiple feature points to represent targets, and then provide a statistical approach to find the most correlated image point pair from different cameras, in order to reach the goal of improving the accuracy of target localization. Note that we focus on $2 \mathrm{D}$ target localization on the ground plane. We assume that the cameras are placed horizontally around a room, which is the most relevant case for many real world applications. Besides, this paper makes the following assumptions about the wireless visual sensor network. Firstly, the location and orientation of each camera node is known within a universal coordinate system. Once a node enters into the networks, its geographical position remains constant. Next, all of the cameras are well calibrated. Finally, all of the nodes are time synchronized.

The initial results of this research have been published in Li et al. (2011), where we briefly introduced our target localization algorithm. In this paper, we expand on that work by providing further insight on the representation of the target by multiple feature points in target localization. In Li et al. (2011), we mainly focus on the single target localization. In this study, we provide a scheme for multiple target localization. The problems of the corresponding target matching and the occlusion between targets are also addressed in multiple target localization without prior knowledge. Besides, we also expend the experiments in a practical environment. Our main contributions include the following: 1) we design a method of using multiple feature points to represent a target in the goal of decreasing the influence of measurement noises and errors on the localization accuracy; 2) we proposed a statistical approach to match the most correlated feature points in different cameras; 3 ) we provide a scheme to address the issue of multiple target localization without prior information. The rest of this paper is organized as follows. Section 2 briefly highlights the related work. Section 3 presents the standard geometrical epipolar model to compute the possible position of single target. Section 4 proposes the technique to represent a target by multiple feature points and the method for finding the correlated point pair by the statistical method. Section 5 studies the case when there is more than one target existing in the field of interest. Section 6 conducts experiments to validate and evaluate the effectiveness of our proposed method and conclusions are given in Section 7.

\section{Related work}

Recently, a lot of researches focus on visual sensor networks, but very limited works related to the target localization in WVSNs has been reported.

Farrell et al. (2009) present a system that uses two cameras to localize the node of wireless sensor networks, and then employs non imaging sensors to estimate the location of targets. Liu et al. (2010) described the common procedure of collaborative single target localization in wireless visual sensor networks, which extracts one feature point and adapts the epipolar geometrical model to compute the target position in the world coordination.
Dios et al. uses Maximum Likelihood technique to fuse the cameras' observations about the location of target. They assume that the target in image can be represented as a single feature point and the level of noise in the measurements is low (Dios et al. 2011). Unfortunately, the target's extraction and localization are always corrupted by measurement noises and errors in practice. Massey et al. (2007) proposes methods to implement target localization using camera networks. They discussed two methods of triangulation for determining a target's position in the global coordinate space, grid based coordination and convex polygon intersection scheme. Their methods heavily rely on an accurate algorithm to define the size, shape, presence, and position of a target within an image. Oztarak et al. (2009) provide a object localization approach which requires the distance between the extracted object and the camera. Unfortunately, the distance information cannot be obtained beforehand in most practical cases. Teng et al. (2014) proposed a method for locating electronic identifiers by integrating the electronic and visual signals. They designed a match engine to find the correspondence between an object's electronic identifier and its visual appearance.

Kulkarni (2007) uses two higher resolution cameras with overlapping coverage to localize an object and compute its Cartesian coordinates. Funiak et al. (2006) proposed a localization algorithm to retrieve the locations and poses of ad hoc placed cameras in a sensor network by tracking a moving object. In their work, they assume that the object appearing in the image of cameras can be represented by a point. Therefore, they do not need to consider the problem of target correspondence. Lin et al. has provided a framework of a camera networks for tracking object in overlapping and non overlapping fields (Lin and Huang, 2011), but they need prior knowledge about the features of an object.

All these methods mentioned above can be regarded as epipolar geometry based solutions. Epipolar geometrical method of computing the coordinate of target is well studied, but it must solve the point correspondence problem because it is very hard to find the correlated image point pairs. Most of the aforementioned works assume that image features required for localization have already been acquired and that the correspondence between the image features are known. Some researchers used Scale Invariant Feature Transform (SIFT) to find feature point correspondences (Sun and Liu, 2011). Since SIFT needs the frame level computation with iterated Gaussian blur operations on images and the frame difference operations on blurred images for feature extraction, its implementation faces challenges of heavy computation, large memory storage and long computational latency (Chiu et al., 2013). Medeiros et al. (2008) and Kurillo et al. (2008) use a LED bar to realize feature points correspondence between cameras. Different from the mentioned works, we present a statistical approach to find the most correlated feature point pair for participating in the task of target localization, without any addi tional tools and prior knowledge of the target.

\section{General target localization model}

In WVSN, since multimedia content, especially video streams, requires transmission bandwidth that is orders of magnitude higher than that supported by currently available sensors (Akyildiz et al., 2007), it is crucial to perform as much local processing (such as compression, error protection, filter, feature extraction, etc.) as possible to reduce the amount of information that needs to be communicated to other nodes (Sanchez Matamoros et al., 2009). In this study, if cameras capture any target, they will extract the target position information from the raw image at local sensor. After wards, they send the position information to the base station where 


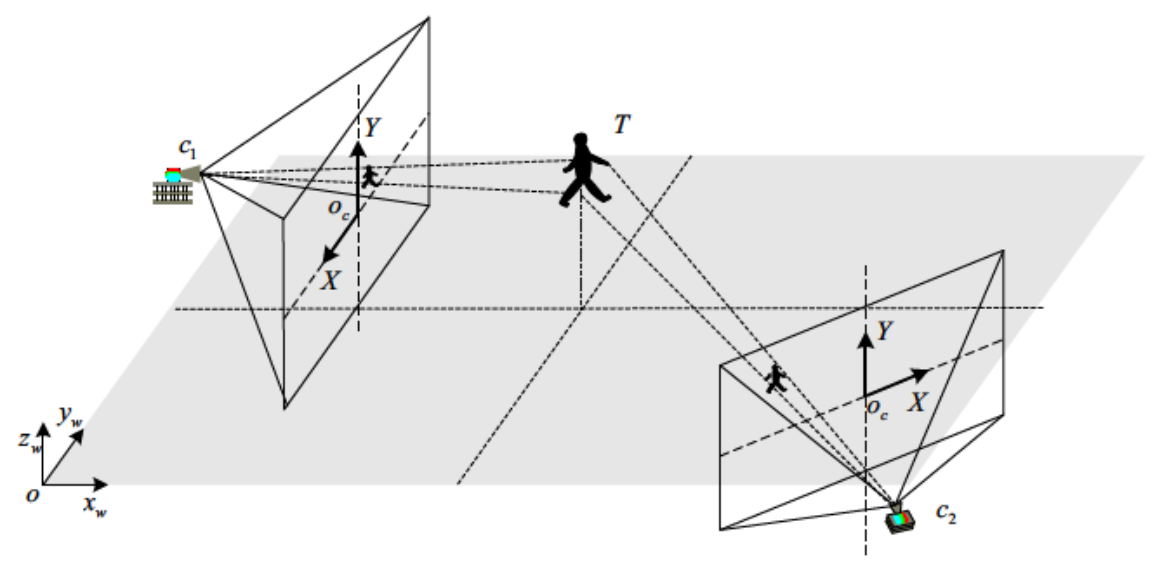

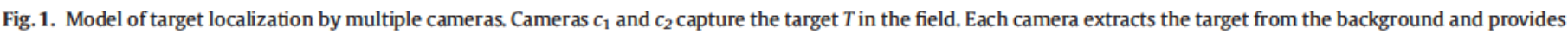
the position information to the base station. In base station, these target position information is fused to generate the coordinate of target in the world frame.

the fusion of target position is executed. Thus, although the accuracy of target position information acquired in the local sensor level is not good enough, with the increase of cameras that are involved in the target localization, it can be gradually improved. The general target localization model in WVSN is described as follows.

Consider a target $T$ in the fixed world coordinate $o x_{w} y_{w} z_{w}$, as shown in Fig. 1. We assume that each camera node in the network is aware of its own location and its orientation in $o x_{w} y_{w} z_{w}$. A camera projects the target $T$ from the world coordinate to its image plane which is denoted as $X o_{c} Y$. For each camera, its optical center can be denoted as $o_{c}$. In this section, it is assumed that only one target is captured by cameras. Note that there is a possibility that more than one target has been captured by WVSN. The method of target localization in the situation with more than one target in the view is discussed in Section 5.

Although each camera node can achieve target detection (i.e. segmenting regions corresponding to the target from the rest of an image), classification(i.e. recognizing the region corresponding to different targets in natural scenes) and tracking (i.e. representing targets by their features such as points, lines or blobs from one frame to another in an image sequence), target localization can only be achieved by fusing the information from multiple camera sensors or by fusing the images taken from one camera at different positions. The reason behind that is that single camera node can only acquire the orientation of the target in the world coordinates. In this paper, the orientation is called the bearing of the target. If the bearings of target from two different images intersected in a point, the point is the possible location of the target in the world coordinate. The principle of target localization is illustrated in Fig. 1.

Specifically, the coordinates of the target can be calculated as follows. Firstly, the target is extracted by the background subtrac tion which is performed at local nodes. Then a feature point is selected to represent the target. As shown in Fig. 2, px and py are the coordinates of the pixel of the feature point on the image plane. We estimate the orientation of the target relative to the camera based on the pixel coordinates in the image. Compared with the orientation of the cameras in the world coordinate, we can compute the bearing of the target in the world coordinates. The bearing of target can be computed according to the following formula:

$k=\tan (\varphi \arctan ((2 p x / p) \cdot \tan (\theta / 2)))$

Where $k$ is the tangent value of the bearing of the target, $\varphi$ is the direction of the camera, $\theta$ is the field of view in horizontal, $p$ is the amount of horizontal pixels of the whole image, and $p x$ is the horizontal pixel coordinate in the image.

We can use the bearings of the target from different cameras that capture the target simultaneously to estimate the target's position.

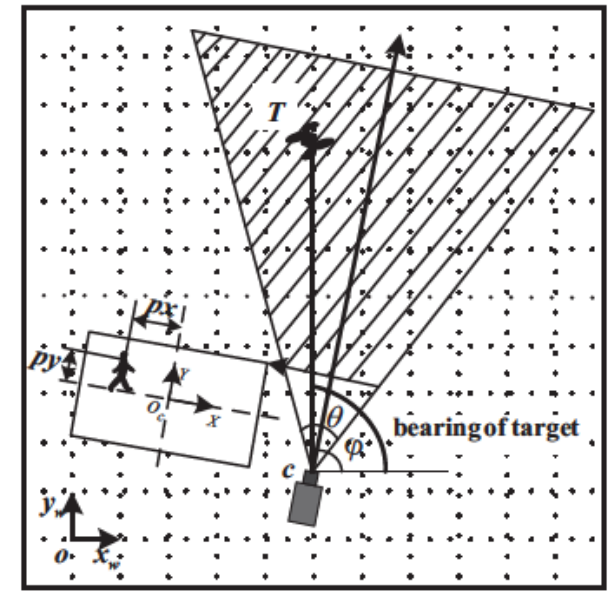

Fig. 2. Geometric model of target localization in the sensor image. Camera project the target from 3D to 2D. The positions of targets can be denoted by pixels, $p x$ and py. Based on the pixels, the bearing of target in the image can be computed.

The computation process is described by the follow equation:

$k_{i}=\frac{y \quad y_{c i}}{x}$

Where $k_{i}$ is the bearing of the target obtained from the $i$ th camera's image. $x_{c}, y_{c i}$ are the coordinates of the $i$ th cameras. The coordinate of the target $(x, y)$ can be computed if we could get at least two different $k_{\dot{p}}$ Note that although a single camera can get two frames with different $k_{i}$ if the camera could move in the field of interest, it is impossible for the case that cameras are fixed at certain positions in the static camera networks. Therefore, the coordinate of the target $(x, y)$ cannot be uniquely estimated by only one camera in the static WVSN. At least two cameras that capture the target should be involved in computing the position of the target. The formula is shown as:

$\left[\begin{array}{ll}k_{1} & 1 \\ k_{2} & 1\end{array}\right]\left[\begin{array}{l}x \\ y\end{array}\right]=\left[\begin{array}{ll}y_{c 1} & k_{1} x_{c 1} \\ y_{c 2} & k_{2} x_{c 2}\end{array}\right]$

Then,

$\left[\begin{array}{l}x \\ y\end{array}\right]=\left[\begin{array}{ll}k_{1} & 1 \\ k_{2} & 1\end{array}\right]^{-1}\left[\begin{array}{ll}y_{c 1} & k_{1} x_{c 1} \\ y_{c 2} & k_{2} x_{c 2}\end{array}\right]$

Thus, we can obtain the position of the target in the world coordinate system. 


\section{Multiple feature points representation in target localization}

In the most existing methods of target localization in WVSN, the target is regarded as a feature point after extraction in images. However, the one feature point extraction approach is invulner able to the additive noise and measurement errors, especially when the cameras' resolutions are not good enough. If the feature point that is corrupted by noises and errors is used in determining the target's coordinates, the accuracy will deteriorates. In this paper, we provide an approach of the target representation by multiple feature points to reduce the influence of noises and errors on the accuracy of target localization.

As mentioned before, the target is extracted by using image processing method at local camera nodes firstly. We assume simple background subtraction (Barnich and Van Droogenbroeck, 2011 ) is performed to extract the hull of the target. We first build a static background frame for representation of the scene without any target. Then we compare a new frame that contains a target with the background frame to detect the region where a signifi cant difference occurs. Thus, the target can be detected. With the background model continuously updating, we can also detect the target in motion by background subtraction. Background subtrac tion method is effective for target extraction, particularly when computation capability is low. Then we want to find correlated point pair to compute the real position of target. To avoid the flaws of the one feature point method which is sensitive to noises and errors, we use multiple feature points to represent the target for being involved in target localization. Then, we use a statistical method to find the feature point that denotes the target position as accurate as possible from the multiple feature points. The multiple feature points ensure the raw data from image contain target position information, and the statistical method guarantees that the feature points which truly reflect the target position are used in the fusion of target localization.

The details of our proposed target localization approach are shown as follows.

\subsection{Step 1}

The hull of the target is extracted by background subtraction method. In order to balance the tradeoff between the accuracy of localization and the cost of computation, two kinds of feature points are chosen to represent the target in images: 1) the center of the hull of target; 2) the intersected point of middle line of the leftmost and the rightmost pixels in horizontal and the middle line of the upmost and the downmost pixels in vertical. Note that if the targets move drastically, more feature points should be selected in the target localization for improving the accuracy.

The center of the hull of target is calculated by the following expression:

$\left\{\begin{array}{l}p x_{\alpha}=\sum_{i=1}^{m} p x_{i} / m \\ p y_{\alpha}=\sum_{i=1}^{m} p y_{i} / m\end{array}\right.$

$p x_{\alpha}$ and $p y_{\alpha}$ are the coordinates of the center of the hull of target. $p x_{i}$ and $p y_{i}$ are the coordinates of the $i$ th pixel on the hull. $m$ is the number of pixels of the target's hull. The position of the other feature point is computed as follows:

$\left\{\begin{array}{l}p x_{\beta}=\frac{p x_{l}+p x_{r}}{2} \\ p y_{k}=\frac{p y_{u}+p y_{b}}{n d 2 p x_{r}} \text { are the coordinates of the leftmost and the right }\end{array}\right.$ most pixels; $p y_{u}$ and $p y_{b}$ are the coordinates of the upmost and the downmost pixels. Note that only $p x_{\alpha}$ and $p x_{\beta}$ are needed to communicate to the base station to make the further decision.

\subsection{Step 2}

We indiscriminately suppose that every feature point is able to represent the position of the target in the image. In order to meet the requirement of one to one correspondence between feature points in different cameras, we select one feature point from each camera to make the corresponding feature point pair group. If $n$ cameras capture the target simultaneously, we would have $2^{n}$ feature point pair groups of the target.

\subsection{Step 3}

For each feature point group, we select two feature points from two different cameras to make the correlated feature point pair Then the possible coordinates of the target can be calculated by the geometrical epipolar model described in Section 4. Each feature point pair group has $C(n, 2)$ pending target coordinates. Then, we compute the means of the target pending position and the mean square errors (MSEs) between the pending positions and the means. The mean of the coordinates of the target is formular ized as follows:

$\left\{\begin{array}{l}X_{j}=\frac{x_{1}+x_{2}+\cdots+x_{n}}{n} \\ Y_{j}=\frac{y_{1}+y_{2}+\cdots+y_{n}}{n}\end{array}\right.$

$X_{j}$ and $Y_{j}$ are the average of the coordinates of the target by the $j$ th feature point pair group; $x_{i}$ and $y_{i}$ are the pending position of target. We can compute the MSE of the target's coordinates of the $j$ th feature point pair group by following expression:

$\operatorname{MSE}_{j}=\frac{\sqrt{\sum_{i=1}^{n}\left(x_{i}-X_{j}\right)^{2}+\sum_{i=1}^{n}\left(y_{i}-Y_{j}\right)^{2}}}{2}$

\subsection{Step 4}

We select a different feature point pair group and execute the computation from step 2 to step 3 . Then we repeat the process until all the feature point pair groups are utilized.

\subsection{Step 5}

From the perspective of statistics, the target's coordinates that are generated by the feature point pair group which truly denotes the target position in the image would converge more compactly in the world coordinates. Thus, we take a comparison of MSEs of the $2 n$ feature point pair groups, and find the minimum one. The mean of the target's positions corresponding to the minimum MSE can be regarded as the finial coordinates of the target in the world reference frame.

\section{Scheme for multiple target localization}

It is possible that there is more than one target existing in the field of interest in practical applications, e.g. battlefield and video surveillance. The biggest difference between single target localiza tion and multiple target localization lies on the corresponding target matching. That is to say that for a target in one image, we have to find the corresponding one in the other images. Besides, we have to consider occlusions by other targets. From the perspective of statistics, the position of the same target estimated by a set of cameras without occlusions would have more 
Table 1

Geometrical parameters of camera and feature points.

\begin{tabular}{llrll}
\hline & Positions $(m)$ & Orientation $(\mathrm{deg})$ & $p x_{\alpha}$ & $p x_{\beta}$ \\
\hline Cam 1 & $(1.0,1.0)$ & 45 & -63 & -59 \\
Cam 2 & $(6.0,0.4)$ & 90 & -93 & -89 \\
Cam 3 & $(9.0,4.0)$ & 180 & 107 & 111 \\
Cam 4 & $(6.2,9.6)$ & 225 & -116 & -123 \\
Cam 5 & $(3.0,9.0)$ & 315 & 82 & 77 \\
Cam 6 & $(0.2,5.0)$ & 0 & -68 & -62 \\
\hline
\end{tabular}

convergence in the world coordinates. Based on that, similar to the method of feature point correspondence in single target localiza tion, we develop a statistical method to match the corresponding target.

Firstly, we extract the visual hulls of targets by background subtraction for every camera, and estimate the polygon centers of the hulls. One target center is selected from each camera that captures targets in order to make the correlated target pair. If $n$ cameras are deployed in the field of interest and one camera captures $m$ targets at most, we could get $m^{n}$ possible correspond ing target pair groups. Then we use the geometrical epipolar model to compute the possible coordinates of the target for each corresponding target pair group. Then we compute the means of the target positions and the MSEs between the estimates and the mean. By the comparisons of the MSEs of the corresponding target pair groups, the group with minimum MSE is set as the accurate correlated pair group of the target. We remove the centers of visual hulls in the corresponding group from the next iteration for determining the other correlated targets. Note that there may be occlusions from other targets in the view of cameras. Thus, we could not get separated visual hulls of the occluded targets in some cameras' images. In this case, the centers of the visual hulls are not removed in the cameras that contain the fewer targets' hulls for the next iteration. The above process is repeated till all the centers are used. By this method, the corresponding target can be matched among the cameras' images. For each corresponding target in the images of cameras, we use the method of single target localization, shown in Section 4, to refine the position of the target in the world coordinates.

\section{Case study and simulations}

\subsection{Evaluation on single target localization}

In this section, we present some simulations to evaluate the performance of our proposed approach. All the simulations in this study have been carried out in MATLAB and Visual $\mathrm{C}++$ on a Pentium machine with 2 GB RAM. For the reason of reducing the simulation complexity, we firstly deployed six camera sensors in a $10 \mathrm{~m} \times 10 \mathrm{~m}$ field. The image format is CIF and the field of view in horizontal is $57.4^{\circ}$. We know cameras' locations and projection matrices with respect to a common world frame, shown in the second and the third columns in Table 1. A target is located in $(4.40 \mathrm{~m}, 6.00 \mathrm{~m})$. The images taken from the six cameras are shown in Fig. 3. Then we use background subtraction method (Barnich and Van Droogenbroeck, 2011) to detect the target, shown in the third row, and then use the Canny edge detector to extract the hull of the target, shown in the last row. According to Eqs. (5) and (6), we can get two feature points of the target. The calculated results are also shown in Table 1. The fourth column shows the horizontal coordinate of the geometry center of the target's hull. The fifth column shows the horizontal coordinates of the middle line of the leftmost and the rightmost border pixels.
If the known parameters of cameras and the feature points information are introduced into Eq. (4), the possible position of the target is obtained. Since there are six cameras deployed in the field of interest and each camera generates two feature points, we can get 64 feature point pair groups. Then using Eqs. (7) and (8), the target position with the minimum MSE can be estimated. In order to verify the effectiveness of the proposed method, we also use the Maximum Likelihood (ML) approach presented in Dios et al. (2011) to estimate the position of the target as a reference. In ML, we assume that the state of the target is measured from $N$ different sensors $z_{1}, \ldots, z_{N}$, where $z_{i}$ is the measurement gathered by sensor $i$. Supposed the measurements of all the sensors can be considered statistically independent, the overall likelihood function can be expressed as the probability of measurement $z$ conditioned to state $x$ :

$\hat{x}=\operatorname{argmax}\{P(z \mid x)\}=\operatorname{argmax}{ }_{x}\left(\prod_{i}^{N} p\left(z_{i} \mid x\right)\right)$

In the study, each measurement is considered to have Gaussian distribution:

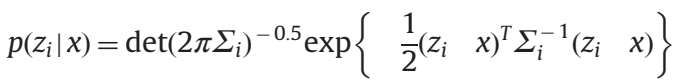

Where $\Sigma_{i}$ is the covariance of measurements from sensor $i$. The ML method estimates the state as the following weighted sum:

$x=\left(\Sigma_{1}^{-1}+\ldots+\Sigma_{N}^{-1}\right)^{-1}\left(\Sigma_{1}^{-1} z_{1}+\ldots+\Sigma_{N}^{-1} z_{N}\right)$

In ML, we assume each camera use one feature point to represent the position of the target in its corresponding image. For a camera $i$, we define the set of cameras which are able to communicate with camera $i$ directly and have the view of the same target as the overlapping camera set of camera $i$. Camera $i$ could generate a possible position of target in world coordinate with each of camera in its overlapping camera set. Then we use the average value of such positions as the measurement of camera $i$, and we assume it contains Gaussian errors with covariance. Thus, the ML model can be used to estimate the location of the target.

We randomly select $M$ cameras out of the six cameras to capture the target. Since it is impossible to compute the MSE by only two cameras, we let the number of cameras $M$ to be selected in the target localization change from 3 to 6 . Fig. 4 plots the errors of target localization using ML approach (red line) and our proposed method (blue line) respectively. It is obviously that localization errors in our proposed approach are less than that in the ML approach. For example, if three cameras capture the target, error between the estimated result and the real position is about $0.52 \mathrm{~m}$ by the ML method. In contrast, by our proposed approach, the error is about $0.44 \mathrm{~m}$. If five cameras capture the target, the error is about $0.19 \mathrm{~m}$ in ML approach. Correspondingly, it is about $0.08 \mathrm{~m}$ in the proposed method. Therefore, this case indicates that our proposed approach has better performance in improving the accuracy of target localization, when the target is captured by the same amount of camera sensors.

Fig. 4 also shows the result that the amount of cameras affects the accuracy of target localization. For example, the accuracy of target localization computed from 4 cameras is better than that produced from 3 cameras in both approaches. That is because the more cameras capture the target, the less influence of error would play on the result. This is also in accordance with the fact that the accuracy of the target localization is gradually improved by involving more cameras. In the ML approach, although there is also improvement of localization accuracy by using multiple cameras, it needs more cameras than our proposed method to reach the same accuracy level. From another perspective, if a certain accuracy level is required, we may need fewer cameras 

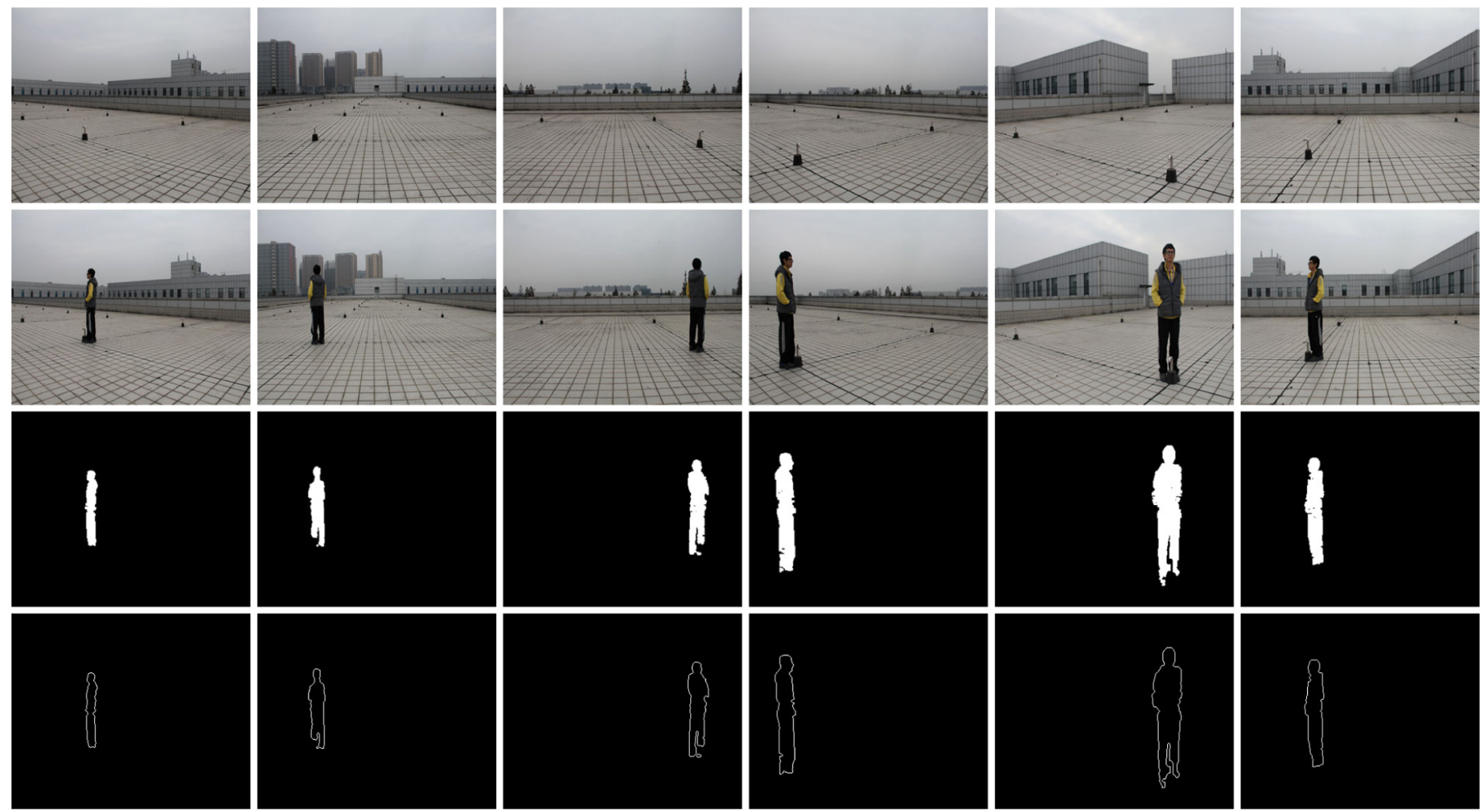

Fig. 3. Images taken from the 6 cameras and the results of background subtractions and contour detection.

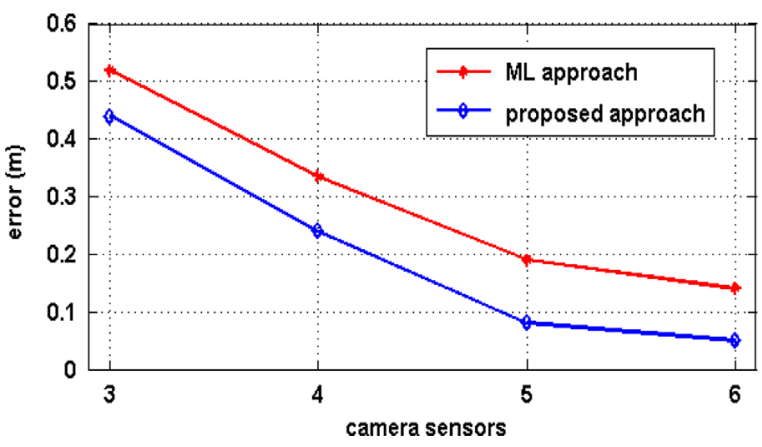

Fig. 4. Error performance of ML approach and the proposed approach. (For interpretation of the references to color in this figure, the reader is referred to the web version of this article).

participating in the task of target localization by the proposed approach. For example, if the users want to obtain the accuracy level of $0.15 \mathrm{~m}$, as shown in Fig. 4, six cameras are needed on average by the ML method, but only five cameras are needed when the proposed approach is used. Therefore, our proposed method has better trade off between localization accuracy and camera sensor usage.

Without loss of generality, our proposed approach is evaluated in a different deployment of camera nodes. The locations and the orientations of cameras are detailed in Table 2. The location of target switches to $(6.5 \mathrm{~m}, 4.5 \mathrm{~m})$. Fig. 5 shows the images taken from the cameras. The positions of feature points are shown in the fourth and the fifth columns of Table 2. The results of target localization by our proposed approach are also compared with that by ML approach, as shown in Fig. 6. Obviously, the proposed approach has better performance in improving the accuracy of target localization than the ML approach. Note that comparing the results in Fig. 4 with that in Fig. 6, we can find that the differences in error level of target localization exist. It is because in each
Table 2

Geometrical parameters of camera and feature points.

\begin{tabular}{lllll}
\hline & Positions $(m)$ & Orientation $(\mathrm{deg})$ & $p x_{\alpha}$ & $p x_{\beta}$ \\
\hline Cam 1 & $(1.5,1.5)$ & 45 & 112 & 117 \\
Cam 2 & $(9.0,2.0)$ & 150 & 83 & 76 \\
Cam 3 & $(6.0,7.5)$ & 270 & -46 & -52 \\
Cam 4 & $(9.0,9.0)$ & 225 & -99 & -103 \\
Cam 5 & $(1.0,9.0)$ & 315 & -26 & -32 \\
Cam 6 & $(2.0,4.0)$ & 30 & 148 & 157 \\
\hline
\end{tabular}

experiment, the cameras have to be calibrated independently, and the noises and measurement errors are random.

Actually, the proposed approach could always have the satisfied performance in improving the accuracy of target localization, as long as the deployment of cameras satisfies two conditions: 1) all the cameras could capture the target, which ensures that the cameras could provide the image the target; and 2) any two of cameras and the target do not lie in a same line, which guarantees that any two of cameras could recover a possible location of the target.

\subsection{Effect of noise on accuracy of target localization}

In order to evaluate the performance of our proposed approach in the environment with additive noise and measurement errors, we artificially add Gaussian noises in the original image of Fig. 3.

Fig. 7 shows the localization errors from the simulation at three different Gaussian noise levels. The means of three noise levels are set to zero, and the standard deviations $\delta$ are set as 0,10 , and 20 respectively. In the Fig. 7 , the three red lines show the localization errors by ML approach, and the three blue lines show the errors by our proposed approach. As can be expected, as noise increases, accuracy of localization by both two approaches decreases, because randomly introduced pixel noise is essentially a scattering 

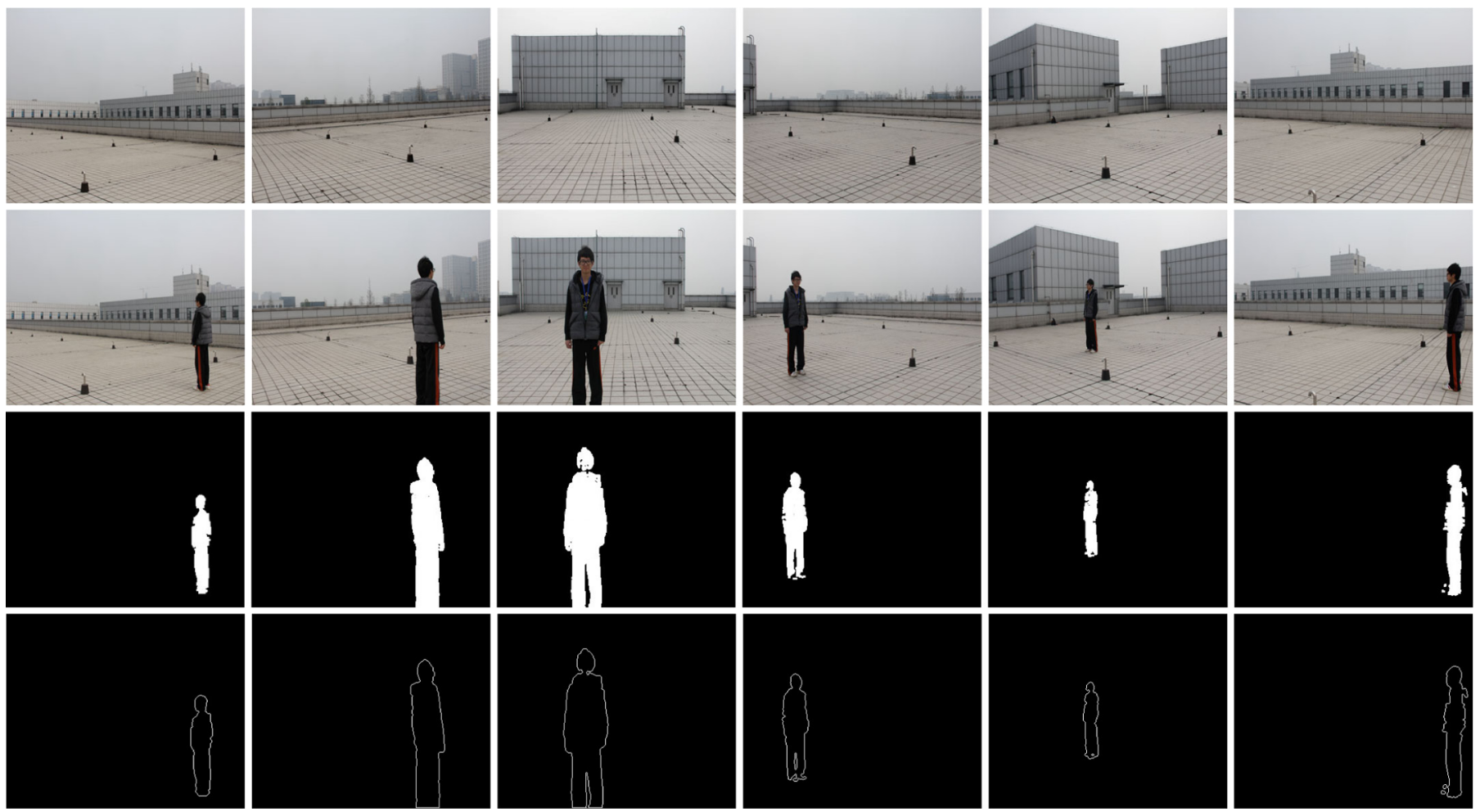

Fig. 5. Images taken from the 6 cameras and the results of background subtractions and contour detection in a different deployment.

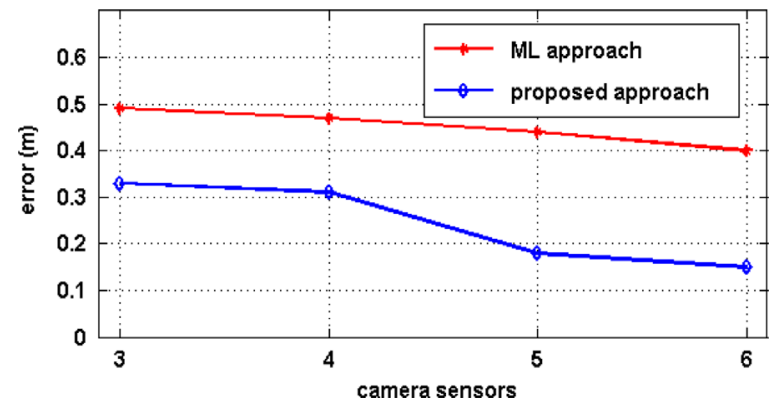

Fig. 6. Errors of ML AND the proposed method in another deployment.

effect on the pixel coordinates of the target. Note that it is apparent that the accuracy by our approach is better than that by ML at any level noises. The localization error in our approach at noise level $\delta=20$ is even less than that in ML at noise level $\delta=0$. The reason behind this is that ML actually belongs to the one feature point approach, which is sensitive to the noises as dis cussed before. Thus, our proposed approach could decrease the influences of measurement errors and noise on the localization accuracy.

\subsection{Performance of multiple target localization}

We also conduct the experiment to testify the effectiveness of our proposed method for locating multiple targets. A real camera network composed of 5 cameras is deployed in a $10 \mathrm{~m} \times 10 \mathrm{~m}$ area. In the area under surveillance, there are two targets that were to be located. The deployments of 5 cameras are shown in Table 3, and the locations of two targets $T_{1}$ and $T_{2}$ are $(6.5 \mathrm{~m}, 4.5 \mathrm{~m})$ and $(4.4 \mathrm{~m}, 3.1 \mathrm{~m})$ respectively. Fig. 8 shows the images of the 5 cameras that capture the two targets and results of target extraction. For locating the two targets respectively, it is required to find the corresponding targets from the 5 cameras. We can

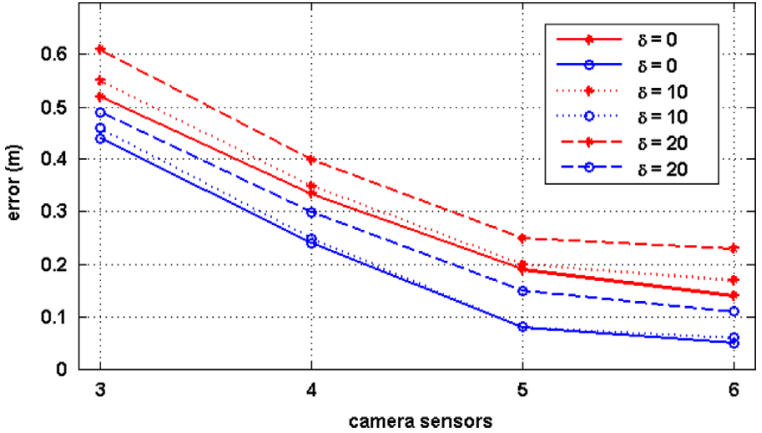

Fig. 7. Evaluations with additive noises. (For interpretation of the references to color in this figure, the reader is referred to the web version of this article).

observe that the contours of the two targets are merged together in Cam1, as shown in the first column in Fig. 8, which increases difficulties of target matching. By our proposed scheme for multi ple targets localization, we indiscriminately suppose that one visual hull just represents one single target, and then we utilize the statistical method to match the corresponding targets among the cameras' images. The localization errors by our proposed method are shown in Fig. 9. After target matching, the localization errors of targets $T_{1}$ and $T_{2}$ are $0.38 \mathrm{~m}$ and $0.30 \mathrm{~m}$ respectively. Then we refine the locations of targets by the method that is provided in Section 4. The errors of two targets decrease to $0.18 \mathrm{~m}$ and $0.16 \mathrm{~m}$ respectively. Thus, we have shown that the proposed method could effectively locate multiple targets in the world coordinates.

\subsection{Evaluation on energy consumption}

In this section, we discuss about the cost of our proposed method. As mentioned in Section 3, one of the major issues in WVSN is the data transmission between the nodes and base station. In our proposed statistical method of target localization, 
since the image is tailored at local nodes, only the position information of feature points is needed to transmit to the base station, which could drastically reduce the bandwidth require ments. For illustrating the cost on energy, we still use the first experiment shown in Fig. 3 as an example. Energy model for communications described in Heinzelman et al. (2002) is applied to specify the energy consumption.

To transmit an $l$ bit message a distance $d$, the radio expends

$E_{T x}(l, d)= \begin{cases}l E_{\text {elec }}+l \epsilon_{f s} d^{2}, & d<d_{0} \\ l E_{\text {elec }}+l \epsilon_{m p} d^{4}, & d \geq d_{0}\end{cases}$

And to receive this message, the radio expends:

$E_{R x}(l, d)=l E_{\text {elec }}$

In our simulation, $E_{\text {elec }}=50 \mathrm{~nJ} / \mathrm{bit}, \epsilon_{f s}=10 \mathrm{pJ} / \mathrm{bit} / \mathrm{m}^{2}, \quad \epsilon_{m p}=$ $0.013 \mathrm{pJ} / \mathrm{bit} / \mathrm{m}^{4}$, and $d_{0}=86.2 \mathrm{~m}$.

We also measure the energy consumption of the contour feature matching scheme (Kim et al., 2011) and the ML scheme. In the contour feature scheme, the silhouette of the target is extracted from the image at local sensor, and the coordinates of all the pixels on the silhouette are needed to transmit to the base station for obtaining the target matching. Due to the fact that the size of the target is not fixed in distinct cameras' images, we take

Table 3

Geometrical parameters of cameras.

\begin{tabular}{llc}
\hline & Positions $(m)$ & Orientation (deg) \\
\hline Cam 1 & $(1.5,1.5)$ & 45 \\
Cam 2 & $(9.0,2.0)$ & 150 \\
Cam 3 & $(6.0,7.5)$ & 270 \\
Cam 4 & $(9.0,9.0)$ & 225 \\
Cam 5 & $(1.0,9.0)$ & 315 \\
\hline
\end{tabular}

the average of amounts of the pixels of the target's silhouette. We firstly study a single hop case between camera nodes and the base station, which means that the extracted target is transmitted from cameras to the base station directly without relays.

If we assume each parameter needs 32 bits to present, the energy consumptions of three schemes in the case of one hop communication can be calculated, as shown in Fig. 10(a). It can be seen that as the distance between the camera and the base station increases, the energy consumptions of all three schemes increase. However, our proposed multiple feature points model requires much less energy for communication than the contour feature scheme. The reason behind is that besides the three parameters about the position and the orientation of a camera, and two parameters about the image's geometry, the horizontal positions of the two feature points' pixel, are needed to transmitted to the base station. Thus, the total bits for transmission is $7 \times 32$ bits in our method. In contrast, the contour feature scheme needs to transmit $111 \times 2 \times 32$ bits to the base station. For the ML method,

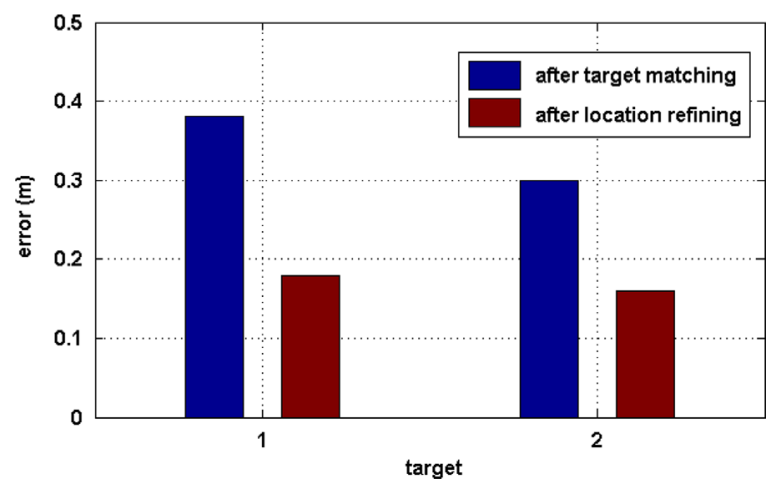

Fig. 9. Errors of targets in multiple target localization.
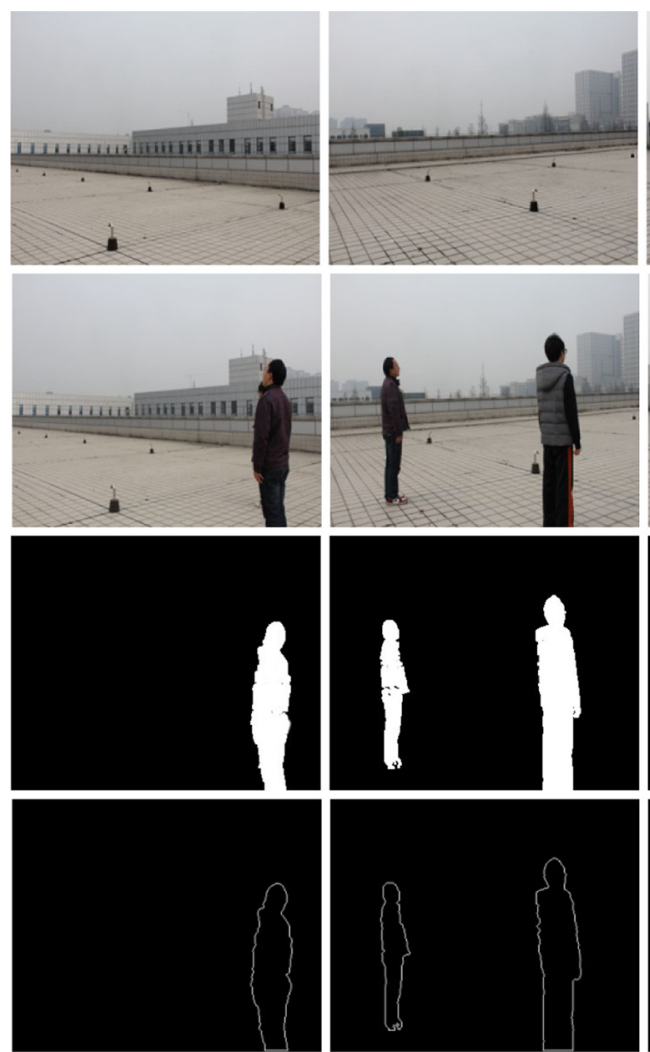
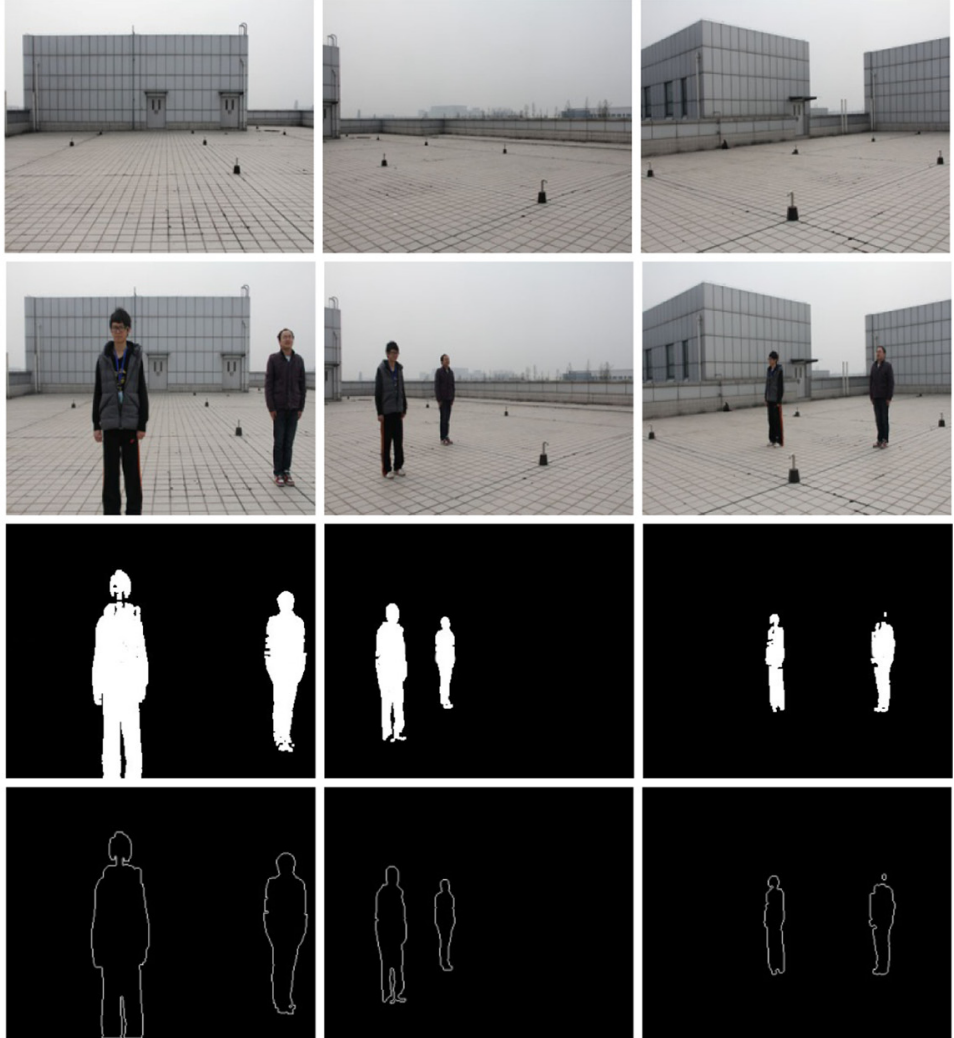

Fig. 8. Image of multiple targets captured by the cameras. 


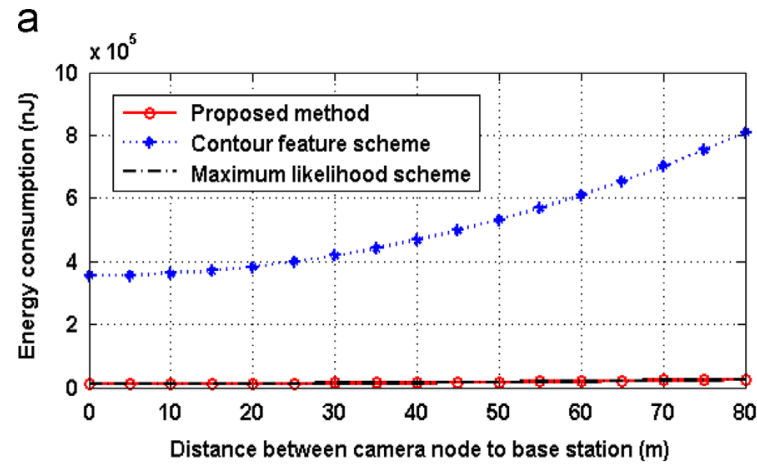

b

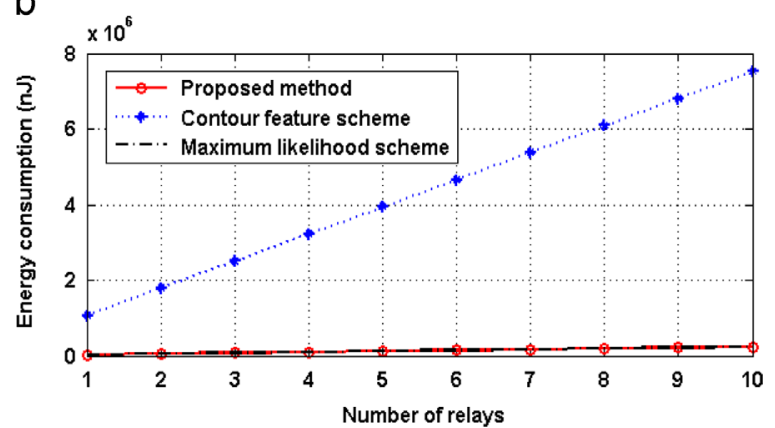

Fig. 10. (a) Energy consumption for communication per node; (b) Energy consumption with relays.

although the only one parameter about the position of the target is needed to transmit to the base station, the information about the covariance of measurements is also required to be transmitted. Thus, the energy consumption level of our proposed method is almost the same as that of the ML method.

If we assume that camera nodes communicate with the base station by using relays, the energy for receiving the message has to be accounted. We study the one route case between cameras and the base station, so that the results are independent of network topologies and specific routing protocols. Thus, energy cost for transmitting $l$ bit message from the source to the base station can be calculated as follows:

$E(l)=E_{T x}\left(l, d_{1}\right)+\sum_{i=2}^{Q}\left(E_{T x}\left(l, d_{i}\right)+E_{R x}\left(l, d_{i}\right)\right)$

where $Q$ is the amount of relays, $d_{i}$ is the distance between nodes at the $i$ th relay. We assume that the distance of every relay equals to $10 \mathrm{~m}$. The results of energy consumption by the three schemes mentioned above are shown in Fig. 10(b). We can observe that the energy consumptions of three schemes increase with the increase of relays. The energy consumption level of the proposed scheme is equal to that of ML scheme, but much lower than that of the contour feature scheme. Thus, we can obtain the conclusion that the proposed method has satisfied performance in energy con sumption for target localization.

\section{Conclusions}

In this paper, we have provided a novel technique for locating targets in wireless visual sensor networks in the goal of improving the accuracy. This technique combines a statistical algorithm with image processing algorithms. In order to reduce the influence of noises and errors on the accuracy, multiple feature points are selected to represent the position of targets. We develop a statistical method to find the most correlated point pair to determine the target's position. Besides, in the case that more than one target exists in the field of interest, we also provide a method to match the corresponding targets. Simulations for locating the single or multiple targets are executed, and results from simulations indicate that our proposed approach could get more desirable localization accuracy and have low communication cost. Besides, in terms of trade off between camera node usage and localization accuracy, our proposed method achieves better performance than the other relative approach.

Future works are oriented to address the problem of target localization in practical congest environment based on the Cookies platform (Krasteva et al., 2011).

\section{Acknowledgments}

This work was supported by National Natural Science Founda tion of China, No. 61403065, Project of Sichuan Science and Technology Bureau, No. 2015JY0084, Fund of Erasmus Mundus TANDAM Project, 2010 and Doctoral Fund of Ministry of Education of China, No. 20120185120034

\section{References}

Akyildiz IF, Melodia T, Chowdury KR. A survey on wireless multimedia sensor networks,. IEEE Wirel Commun 2007;14(6):32-9.

Akyildiz Ian F, Melodia Tommaso, Chowdhury Kaushik R. A survey on wireless multimedia sensor networks. Comput Netw 2007:51:921-60.

Barnich O, Van Droogenbroeck M. ViBE: a universal background subtraction algorithm for video sequences. IEEE Trans Image Process 2011;20(6):1709-24.

Charfi Youssef, Wakamiya Naoki, Murata Masayuki. Challenging issues in visual sensor networks,. IEEE Wirel Commun 2009;16(2):44-9.

Chiu LC, Chang TS, Chen JY, Chang NYC. Fast SIFT design for real-time visual feature extraction. IEEE Trans Image Process 2013;22(8):3158-67.

Dai R, Akyildiz IF. A spatial correlation model for visual information in wireless multimedia sensor networks. IEEE Trans Multimed 2009;11(6):1148-59.

Dios JRM, Gonzales AJ, Ollero A. Chapter 3: Localization and tracking using camerabased wireless sensor networks. 2011.

Ercan D Yang, Gamal AE, Guibas L. Optimal placement and selection of camera network nodes for target localization. DCOSS; 2006. p. 389-404.

Farrell R, Garcia R, Lucarelli D, Terzis A, Wang I-J. Target localization in camera wireless networks.. Pervasive Mob Comput 2009;5(2):S165-81.

Funiak S, Guestrin C, Paskin M, Sukthankar R. Distributed localization of networked cameras. In: Proceedings of the fifth international conference on information processing in sensor networks. IPSN; 2006. p. 34-42.

Heinzelman WB, Chandrakasan AP, Balakrishnan H. An application-specific protocol architecture for wireless microsensor networks. IEEE Trans Wirel Commun 2002;1(4):660-70.

Jiang $\mathrm{F}, \mathrm{Hu}$ J. Cooperative multi-target tracking in passive sensor-based networks. In: Proceedings of IEEE wireless communications and networking conference. WCNC; 2013, p. 4340-45.

Teng Jin, Zhang Boying, Zhu Junda, Li Xinfeng, Xuan Dong, Zhang YuanF. EV-Loc: integrating electronic and visual signals for accurate localization. IEEE/ACM Trans Netw 2014;22(4):1285-96.

Kim T, Lee S, Paik J. Combined shape and feature-based video analysis and its application to non-rigid object tracking. IET Image Process 2011;5(1):87-100.

Krasteva Y, Portilla J, de la Torre E, Riesgo T. Embedded run-time reconfigurable nodes for wireless sensor networks applications. IEEE Sens J 2011;11(9):1800-10.

Kulkarni Purushottam. Senseye: a multi-tier heterogeneous camera sensor network. Amherst, MA: University of Massachusetts; 2007.

Kurillo G, Li Zeyu, Bajcsy R. Wide-area external multi-camera calibration using vision graphs and virtual calibration object. In: Prooceedings of the second ACM/IEEE international conference on distributed smart cameras. Stanford (USA); December 2008. p. 1-9.

Li W, Zhang W. Sensor selection for improving accuracy of target localization in wireless visual sensor networks. IET Wirel Sens Netw 2012;2(4):293-301.

Li Wei, Portilla Jorge, Moreno Felix, Liang Guixuan, Riesgo Teresa. Improving target localization accuracy of wireless visual sensor networks. In: Proceedings of the 37th IEEE industrial electronics conference. Melbourne; 7-9 November 2011.

Lin DT, Huang KY. Collaborative pedestrian tracking and data fusion with multiple cameras,. IEEE Trans Inf Forensics Secur 2011;6(4):1432-44.

Liu Liang, Zhang Xi, Ma Huadong. Optimal node selection for target localization in wireless camera sensor networks. IEEE Trans Veh Technol 2010:59(7):3562-76.

Mao Guoqiang, Fidan Baris, Anderson Brian DO. Wireless sensor network localization techniques. Comput Netw 2011;51(10):2529-53.

Massey T, Kapur R, Dabiri F, Vu LN, Sarrafzadeh M. Localization using lowresolution optical sensors. In: Proceedings of the 4th IEEE international conference on mobile ad-hoc and sensor systems. Italy; 2007. 
Medeiros H, Iwaki H, Park J. Online distributed calibration of a large network of wireless camera using dynamic clustering. In: Proceedings of ACM/IEEE international conference on distributed smart cameras; 2008.

Oztarak H, Akkaya K, Yazici A. Lightweight object localization with a single camera in wireless multimedia sensor networks. In: Proceedings of IEEE global telecommunications conference. Hawaii (USA); December 2009.

Sanchez-Matamoros JM, Martinez-de Dios JR, Ollero A. Cooperative localization and tracking with a camera-based WSN. In: Proceedings of the 2009 IEEE international conference on mechatronics. Spain; April 2009.
Soro Stanislava, Heinzelman Wendi. A survey of visual sensor networks. Adv Multimed 2009;2009:1-21.

Sun Li, Liu Guizhou. Visual object tracking on combination of local description and global representation. IEEE Trans Circuits Syst Video Technol 2011;21 (4):408-20. 